\title{
Association of ${ }^{11} \mathrm{C}$-Methionine PET uptake with site of failure after concurrent temozolomide and radiation for primary glioblastoma multiforme
}

\author{
Irwin H. Lee ${ }^{1}$, Morand Piert ${ }^{2}$, Diana Gomez-Hassan ${ }^{3}$, Larry Junck ${ }^{4}$, Lisa Rogers ${ }^{4}$, James \\ Hayman ${ }^{1}$, Randall K Ten Haken ${ }^{1}$, Theodore S. Lawrence ${ }^{1}$, Yue Cao ${ }^{1}$, and Christina Tsien ${ }^{1}$ \\ 1 Department of Radiation Oncology, University of Michigan \\ 2 Department of Nuclear Medicine, University of Michigan \\ 3 Department of Radiology, University of Michigan \\ 4 Department of Neurology, University of Michigan
}

\begin{abstract}
Purpose-To determine if increased uptake on 11C-methionine-PET (MET-PET) imaging obtained prior to radiation therapy and temozolomide is associated with the site of subsequent failure in newly diagnosed glioblastoma multiforme (GBM).

Methods-Patients with primary GBM were treated on a prospective trial with dose-escalated radiation and concurrent temozolomide. As part of the study, MET-PET was obtained prior to treatment but was not used for target volume definition. Using automated image registration, we assessed whether the area of increased MET-PET activity (PET-GTV) was fully encompassed within the high-dose region and compared the patterns of failure for those with and without adequate highdose coverage of the PET-GTV.
\end{abstract}

Results-Twenty-six patients were evaluated with a median follow-up of 15 months. Nineteen of 26 had appreciable $\left(>1 \mathrm{~cm}^{3}\right)$ volumes of increased MET-PET activity prior to treatment. Five of nineteen patients had PET-GTV that was not fully encompassed within the high-dose region, and all five patients had non-central failures. Among the 14 patients with adequately covered PET-GTV, only 2 had non-central treatment failures. Three of 14 patients had no evidence of recurrence with over 1 year after radiation therapy. Inadequate PET-GTV coverage was associated with increased risk of non-central failures. ( $\mathrm{p}<0.01)$.

Conclusion-Pretreatment MET-PET appears to identify areas at highest risk for recurrence for patients with GBM. It would be reasonable to test a strategy of incorporating MET-PET into radiation treatment planning, particularly for identifying areas for conformal boost.

\footnotetext{
Corresponding author: Christina Tsien, M.D, University of Michigan Medical Center, Dept. of Radiation Oncology, 1500 E. Medical Center Drive, Ann Arbor, Michigan 48109, Tel: 734-936-4288, Fax: 734-763-7370, Email: ctsien@ umich.edu.

Presented at ASTRO 2007 Annual meeting (October 28-November 1, 2007, Los Angeles, CA, USA)

Publisher's Disclaimer: This is a PDF file of an unedited manuscript that has been accepted for publication. As a service to our customers we are providing this early version of the manuscript. The manuscript will undergo copyediting, typesetting, and review of the resulting proof before it is published in its final citable form. Please note that during the production process errors may be discovered which could affect the content, and all legal disclaimers that apply to the journal pertain.
} 


\section{Introduction}

Glioblastoma multiforme (GBM) is the most common primary malignancy of the adult central nervous system and carries with it an exceptionally poor prognosis. Although radiation therapy has been shown to prolong survival, patients treated with radiation therapy typically progress within the radiation field[1-3]. The addition of concurrent and adjuvant temozolomide (TMZ) to standard-dose radiation (60 Gy) improves survival, but most patients continue to develop local failures[4]. Thus, further intensification of local therapy may be needed in conjunction with new chemotherapeutic agents like TMZ.

Prior dose-escalation studies with radiation alone suggest that patterns of failure can be altered with sufficiently high doses of radiation. Using the traditional dose of $60 \mathrm{~Gy}$, approximately $80 \%$ of failures occur "centrally," i.e., within $2 \mathrm{~cm}$ of the gross tumor [3,5]. By comparison, in a single-institution study using accelerated proton boost to 90 cobalt gray equivalent (CGE), only $1 / 23$ patients was found to have recurrent tumor within the 90 CGE volume [6]. In a series of patients treated with combined external beam radiation and interstitial brachytherapy boost, Cox proportional hazards analysis showed that increased dose was associated with a lower local failure rate [7]. Similarly, in a series of patients treated with external beam radiation followed by a stereotactic radiosurgical (SRS) boost, the rate of failures within $2 \mathrm{~cm}$ of the SRS volume was only $38 \%$ [8]. Finally, a retrospective series of patients treated with high-dose conformal radiation compared to historical controls showed a relative decrease in the proportion of local failures[9]. Thus, there is evidence to suggest that high-dose conformal radiation may provide improved local control. However, this apparent increase in local control is also associated with a higher rate of toxicity. Patients from both the brachytherapy and SRS boost trials had higher rates of symptomatic radiation necrosis in comparison to patients treated to the current standard of $60 \mathrm{~Gy}$.

The dose-escalation efforts described above have relied on standard contrast-enhanced MRI for defining the extent of disease, and thus the high-dose radiation was delivered to the entire contrast enhancing volume plus margin. The results of these studies suggest that treating large volumes to high doses leads to unacceptable complication rates with symptomatic radiation necrosis in $50 \%$ of patients or more. Therefore, better imaging techniques may be needed to identify target volumes that are at the highest risk for recurrence, so the high-dose volume might be reduced in size.

Metabolic imaging studies such ${ }^{11} \mathrm{C}$-methionine PET (MET-PET) may improve our ability to identify target volumes at highest risk of local failure. MET-PET imaging demonstrates increased metabolic activity due to increased amino-acid transport in glioma cells compared to normal brain[10]. In comparison, FDG-PET for target definition is complicated by the high level of intrinsic glucose uptake in the brain[11]. There is evidence that MET-PET may be useful for identifying residual tumor after resection and in identifying recurrent gliomas[12, 13]. However, no prospective studies have assessed whether MET-PET prior to treatment may be used to predict sites of subsequent treatment failures.

Therefore, we initiated a phase I/II radiation dose-escalation trial to determine the maximum tolerated dose (MTD) of RT when given with concurrent TMZ for newly diagnosed GBM. We hypothesized that areas of increased MET-PET activity would be at higher risk for recurrence. To test this hypothesis, we prospectively acquired MET-PET scans to compare initial areas of increased methionine uptake with subsequent site of failure. 


\section{Materials and Methods}

\section{Study patients}

This study was reviewed and approved by the University of Michigan's Institutional Review Board. From November 2003 through October 2006, thirty patients were enrolled on the protocol. Eligibility criteria included: histologically-confirmed supratentorial WHO grade IV gliomas, age at least 18 years, Karnofsky performance status at least 70, minimal life expectancy of 12 weeks, and adequate bone marrow, liver, and renal function. Exclusion criteria included: multifocal or recurrent gliomas, involvement of the brainstem or posterior fossa, CSF dissemination, severe concurrent disease, prior history of radiation therapy that would lead to overlap with new fields, or inability to undergo MRI. Treatment was required to be initiated within 5 weeks of surgical resection or biopsy.

\section{Treatment}

Patients were treated with concurrent TMZ $\left(75 \mathrm{mg} / \mathrm{m}^{2}\right.$ daily) and dose-escalated intensitymodulated radiation therapy (IMRT) using a simultaneous boost delivered in 30 treatments over 6 weeks. Treatment planning CT was obtained with the patient immobilized in an individualized thermoplastic mask. MET-PET was obtained before treatment but not used to define the radiation treatment volume. Post-operative MRI scans with contrast were coregistered to the treatment planning CT and all other imaging studies. Gross target volumes (GTV) were defined as the residual gross tumor or resection cavity, based on the contrastenhancing T1 weighted images. GTVs were then expanded uniformly by $1.5 \mathrm{~cm}$ to form the clinical target volumes (CTV). The CTV and GTV were then expanded uniformly by $0.5 \mathrm{~cm}$ to generate planning target volumes, PTV1 and PTV2, respectively (Figure 1). Forward- or inverse-planned IMRT plans were generated to deliver $60 \mathrm{~Gy}$ to PTV1 and a higher dose (range: 66-81 Gy) to PTV2, with at least $99.5 \%$ of the PTV encompassed by the $95 \%$ prescription isodose surface (IDS).

\section{Follow-up and Failure Definition}

Patients were seen in follow-up at 1 month, then every 2-3 months for 1 year, and every 3-4 months thereafter. Standard contrast-enhanced and non-enhanced MRI was obtained in conjunction with clinical follow-up. Progression was defined as $>25 \%$ increase in greatest cross-sectional area of contrast-enhancement on MRI or new contrast-enhancing lesions on MRI beyond the initial tumor. MRI changes occurring 1-3 months following radiation therapy that were accompanied by neither clinical symptoms nor increased need for steroids were carefully followed but not categorized as tumor progression due to the possibility of pseudoprogression[14]. Patients suspected of having disease progression generally underwent repeat MRI one month later for confirmation. Magnetic resonance spectroscopy (MRS) and METPET scans were obtained at the time of suspected tumor progression for further confirmation. If there were uncertainty in distinguishing tumor recurrence from radiation necrosis, image guided re-excision or biopsy of the suspicious region was performed whenever possible. Patients who underwent re-excision or biopsy were considered to have treatment failure if there were any histological evidence of residual or recurrent disease.

\section{MET-PET Scan}

MET-PET was obtained on a Siemens ECAT EXACT HR+ whole body tomograph (axial resolution $4.1 \mathrm{~mm}$ FWHM in the center of the field of view) [15]. Emission scan image data obtained in 3D mode between 10 and 30 minutes following intravenous injection of approximately $740 \mathrm{MBq}$ of MET were summed into a single frame and analyzed. Prior to MET injection, a transmission scan of 6 min duration (approximately 1.5 million counts) was acquired using three Ge-68 rod sources. Emission data corrected for attenuation, scatter, and 
random coincidences were reconstructed iteratively using an All pass filter with 4 iterations and 16 subsets ( $128 \times 128$ pixel matrix).

The MET uptake was normalized by the mean cerebellar uptake. PET-GTV was automatically segmented using a threshold of 1.5 times the mean cerebellar uptake[16]. Uptake in normal tissues outside normal brain including lacrimal and pituitary glands was excluded manually.

\section{Image and Radiation Dose Co-registration}

The pre-treatment MET-PET scan and treatment planning CT scan were co-registered with the pre-treatment diagnostic MRI using functional imaging analysis tools (FIAT), a software package developed at the University of Michigan that includes a mutual information algorithm [17]. MRI at the time of tumor progression was also registered to the pre-treatment diagnostic MRI. Registration of the accumulated radiation dose plan with these imaging series was accomplished by applying (to the dose distribution) the same transformation that resulted from registration of the treatment planning CT. In each case,registration accuracy was verified using a combination of methods, including split-screen, scrolling slice by slice in different planes, as well as comparing contour overlays. This ensured that accurate image registration was obtained for different scans despite small changes in head position or image slice orientation. These methods are able to detect discrepancies in image registration with a magnitude of 1 voxel or approximately $3-5 \mathrm{~mm}$.

\section{Analysis of Patterns of Failure and PET-GTV Coverage}

All patients had repeat imaging at the time of treatment failure. Following image coregistration, a recurrence volume of interest (rVOI) was defined by a neuro-radiologist and radiation oncologist as the contrast-enhancing region on MRI at the time of tumor progression. Large central areas that were non-enhancing were excluded from the rVOI. The percentage of rVOI enclosed by the 95\% (high-dose prescription) isodose surface was determined in order to classify the failure as: central (>95\% covered), in-field (> 80-95\%), marginal (20-80\%), or distant $(<20 \%)$ [18]. Similarly, we determined the fraction of the PET-GTV encompassed by the $95 \%$-isodose surface with "adequate" coverage being defined as more than $95 \%$ of the PET-GTV volume encompassed within the $95 \%$-isodose surface.

\section{Results}

\section{Patient Characteristics and Outcomes}

At the time of this analysis (last updated September 10, 2007), 30 patients had been enrolled on study with a minimum follow-up of 1 year for those who had not progressed. Among these, two patients died from aplastic anemia, and 1 patient died of sepsis within 3 months of completing radiation. These patients could not be evaluated with regard to treatment failure and were excluded from the patterns of failure analysis. In addition, one patient did not have MET-PET prior to treatment and was also excluded from this analysis. Thus, a total of 26 patients with pre-treatment MET-PET scans were analyzed for patterns of failure with a median follow-up of 15 months (Table 1). 13 patients had gross total resection (GTR), seven patients had subtotal resection (STR), and six patients had stereotactic biopsy. Median progression free survival (PFS) from the date of enrollment for the analyzed population was 8 months (range: $1.5-20$ months).

Among the 26 patients analyzed, three patients remain alive with no clinical or radiological evidence of progression with more than one year of follow-up. Ten patients underwent reresection or stereotactic biopsy to confirm tumor progression to determine the most appropriate treatment course; one patient underwent autopsy. Among these eleven patients in whom tissue was obtained after progression, pathologic review revealed one patient to have only radiation 
necrosis at time of re-resection and no evidence of recurrent tumor; the other 10 patients were found to have some evidence of recurrent or residual GBM, including 3 cases with extensive areas of radiation effect and vasculopathy in addition to the residual/recurrent disease. Twelve patients were judged to have progression by clinical and radiological criteria without pathologic confirmation; among these, 8 have expired (including 1 patient who progressed while undergoing radiation therapy and received only $20 \mathrm{~Gy}$ ) and 4 remain alive11-19 months after progression. Although the majority of patients who did not undergo re-resection died within 6 months following documented tumor progression, four of these patients have responded to second-line chemotherapy and remain alive one year following clinical diagnosis of tumor progression.

\section{Methionine PET-GTV Characteristics}

The median PET-GTV size with a lesion-to-cerebellum ratio of 1.5 or above was $5.5 \mathrm{~cm}^{3}$ (range: $0.3-43.8 \mathrm{~cm}^{3}$ ) and varied inversely with the reported extent of resection (Figure 2). Among the 13 patients reported to have GTR, seven patients had less than $1 \mathrm{~cm}^{3}$ of increased PET activity. The maximum PET-GTV in the GTR group was $5.5 \mathrm{~cm}^{3}$. By contrast, the PETGTV in patients who underwent stereotactic biopsy ranged from 12.1 to $43.8 \mathrm{~cm}^{3}$.

We began by assessing the relationship between the area of increased MET-PET uptake and the contrast-enhancing region noted on pretreatment MRI. The size of the PET-GTV was typically smaller than the contrast-enhancing volume on MRI (mean $11.5 \mathrm{vs} 18.4 \mathrm{~cm}^{3}, \mathrm{p}<$ 0.01 , paired t-test). In the majority of the stereotactic biopsy cases, the region of increased PET activity appeared similar to the contrast-enhancing lesion. However, there were cases in which the PET-GTV extended beyond the contrast-enhancing region (Figure 3A). In patients who underwent surgical resection, MET-PET was particularly helpful in establishing the extent of residual tumor since post-operative changes could not always be distinguished from residual enhancement on MRI (Figure 3B).

\section{MET-PET Activity and Patterns of Failure}

To assess the potential utility of MET-PET in identifying regions at high risk for recurrence, we compared the patterns of failure for those patients whose PET-GTV was fully encompassed by the 95\% IDS to those whose PET-GTV extended beyond the 95\% IDS (Table 2). We hypothesized that if MET-PET were useful in addition to MRI in detecting regions at highest risk, then there would be significantly more non-central failure in patients whose PET-GTV was fully covered. The seven patients with no appreciable MET-PET activity (i.e., PET-GTV $<1 \mathrm{~cm}^{3}$ ) were omitted from this analysis.

In five patients, the PET GTV was not fully encompassed by the 95\% IDS, and all 5 of these patients recurred with non-central failures. In comparison, only two of 14 patients whose PETGTV was fully encompassed by the 95\% IDS had non-central failures. This includes 3 patients that have not yet failed with over 12 months of followup. Using the Fisher exact test, this apparent association between suboptimal coverage of the PET-GTV and subsequent noncentral failure was statistically significant $(\mathrm{p}<0.01)$.

Review of the 5 cases in which the PET-GTV was inadequately covered revealed one case in which a distinct nodule was noted on the pre-treatment MET-PET which was not apparent on contrast-enhanced MRI. This patient developed a recurrence one month after completing radiation treatment in the area of MET-PET uptake (Figure 4a). Minimum dose to the nodule with increased PET uptake was $43 \mathrm{~Gy}$. In the other four patients, the minimum dose to the PET-GTV was at least $58 \mathrm{~Gy}$, the minimum dose to PTV1. In these remaining cases, the relationship between PET-GTV and subsequent site of failure was less obvious, but there was 
still overlap noted between the areas of initial increased PET uptake and the eventual area of recurrence on MRI (Figure 4b).

\section{Discussion}

In this study, we have found that MET-PET provides potentially important additional information over standard anatomic imaging with contrast-enhanced MRI. There was a statistically significant correlation between the presence of increased MET-PET uptake outside the high-dose region and subsequent non-central failure. This represents the first prospective study in which pretreatment MET-PET imaging was compared with subsequent patterns of failure in GBM patients treated with radiation and TMZ. These findings suggest that it would be reasonable to test a strategy of using MET-PET to identify focal areas for a conformal radiation boost.

The observation that regions of increased MET-PET activity and of contrast-enhancement on MRI are imperfectly correlated is consistent with the findings of a prior study in which patients with primary GBM underwent MET-PET prior to undergoing radiation[13]. In that study, which was limited to patients who had undergone subtotal resection, $87 \%$ of patients were found to have discrepancies between the Gd-enhancing volume and areas of increased PET activity. However, that study did not include data on subsequent failures, so it was unclear whether the MET-PET activity would be important in identifying regions at high risk for progression.

In this limited dataset, we have found not only that MET-PET reveals areas of abnormality that are not apparent by contrast-enhancement on MRI but also that there is a correlation between the location of increased pre-radiation MET-PET activity and the subsequent site of failure. All patients who had suboptimal coverage of the PET-GTV had non-central failures whereas only 2 of 14 patients with adequately covered PET-GTV had non-central failures. By focusing on whether the initial MET-PET activity fell entirely within the high-dose region and whether the recurrences fell within the high-dose region, we have circumvented some of the challenges associated with trying to localize the origin of failure more precisely. The correlation between the pattern of failure and the adequacy of coverage of the pretreatment MET-PET supports the incorporation of MET-PET into radiation treatment planning, although additional data are clearly needed to corroborate our findings.

In addition to MET-PET, other modalities may also be useful in identifying regions at increased risk for progression. In particular, recently published studies have shown that 3D-magnetic resonance spectroscopic imaging (MRSI) may serve as an adjunct to conventional MRI in delineating the extent of disease in high-grade gliomas and that combining standard MRI with MRSI volumes leads to improved coverage of areas that subsequently progress[19-21]. Other novel MR techniques, including perfusion and diffusion-weighted MRI obtained prior to and during treatment, have been demonstrated to be predictive of outcomes, but additional work is needed to determine if these techniques can specifically identify areas within the target that are at risk for failure[22,23]. In the future, it seems likely that the complementary information obtained with multiple imaging modalities will lead to the most efficient delineation of the appropriate target volume.

One limitation of this study is the small number of subjects enrolled. However, the purpose of the study was to evaluate prospectively whether MET-PET might be useful for treatment planning purposes, and the correlation we found between the location of increased PET activity and the subsequent pattern of failure in this preliminary dataset did achieve statistical significance and provide justification for additional work on the use of MET-PET for treatment planning. Another potential criticism of our study is that only a subset of the patients in this 
study had histological confirmation of treatment failure. However, given that our policy was to obtain tissue in cases with uncertainty and that 10 of 11 patients with additional tissue were found to have evidence of recurrence, it seems likely that nearly all of the patients deemed to have progression on clinical and imaging criteria would have had positive biopsies.

Based on the results of our study, we believe it would be reasonable to design a prospective trial testing whether selectively increasing the radiation dose to MET-PET avid regions could improve the outcome of treatment. By limiting the radiation boost volume, higher doses may be safely achieved, so focal dose-escalation may be more successful than uniformly escalating dose across the entire contrast-enhancing MRI volume. The addition of TMZ may be especially effective as a radiation sensitizer if the radiation dose to normal brain can be limited by better targeting. If new, targeted chemotherapy agents lead to further improvements in control of microscopic disease, radiation can be used primarily to control disease in limited regions that have the highest risk of progression, i.e., where those agents are most likely to fail.

\section{Acknowledgements}

NIH 3PO1 CA 59827

\section{References}

1. Aydin H, Sillenberg I, von Lieven H. Patterns of failure following CT-based 3-D irradiation for malignant glioma. Strahlenther Onkol 2001;177(8):424-31. [PubMed: 11544905]

2. Garden AS, Maor MH, Yung WK, et al. Outcome and patterns of failure following limited-volume irradiation for malignant astrocytomas. Radiother Oncol 1991;20(2):99-110. [PubMed: 1851573]

3. Wallner KE, Galicich JH, Krol G, et al. Patterns of failure following treatment for glioblastoma multiforme and anaplastic astrocytoma. Int J Radiat Oncol Biol Phys 1989;16(6):1405-9. [PubMed: 2542195]

4. Stupp R, Mason WP, van den Bent MJ, et al. Radiotherapy plus concomitant and adjuvant temozolomide for glioblastoma. N Engl J Med 2005;352(10):987-96. [PubMed: 15758009]

5. Chang EL, Akyurek S, Avalos T, et al. Evaluation of peritumoral edema in the delineation of radiotherapy clinical target volumes for glioblastoma. Int J Radiat Oncol Biol Phys 2007;68(1):14450. [PubMed: 17306935]

6. Fitzek MM, Thornton AF, Rabinov JD, et al. Accelerated fractionated proton/photon irradiation to 90 cobalt gray equivalent for glioblastoma multiforme: results of a phase II prospective trial. J Neurosurg 1999;91(2):251-60. [PubMed: 10433313]

7. Sneed PK, Lamborn KR, Larson DA, et al. Demonstration of brachytherapy boost dose-response relationships in glioblastoma multiforme. Int J Radiat Oncol Biol Phys 1996;35(1):37-44. [PubMed: 8641924]

8. Shrieve DC, Alexander E 3rd, Black PM, et al. Treatment of patients with primary glioblastoma multiforme with standard postoperative radiotherapy and radiosurgical boost: prognostic factors and long-term outcome. J Neurosurg 1999;90(1):72-7. [PubMed: 10413158]

9. Tanaka M, Ino Y, Nakagawa K, et al. High-dose conformal radiotherapy for supratentorial malignant glioma: a historical comparison. Lancet Oncol 2005;6(12):953-60. [PubMed: 16321763]

10. Jager PL, Vaalburg W, Pruim J, et al. Radiolabeled amino acids: basic aspects and clinical applications in oncology. J Nucl Med 2001;42(3):432-45. [PubMed: 11337520]

11. Grosu AL, Piert M, Weber WA, et al. Positron emission tomography for radiation treatment planning. Strahlenther Onkol 2005;181(8):483-99. [PubMed: 16044216]

12. Grosu AL, Weber WA, Franz M, et al. Reirradiation of recurrent high-grade gliomas using amino acid PET (SPECT)/CT/MRI image fusion to determine gross tumor volume for stereotactic fractionated radiotherapy. Int J Radiat Oncol Biol Phys 2005;63(2):511-9. [PubMed: 16168843]

13. Grosu AL, Weber WA, Riedel E, et al. L-(methyl-11C) methionine positron emission tomography for target delineation in resected high-grade gliomas before radiotherapy. Int J Radiat Oncol Biol Phys 2005;63(1):64-74. [PubMed: 16111573] 
14. Taal W, Brandsma D, de Bruin HG, et al. The incidence of pseudo-progression in a cohort of malignant glioma patients treated with chemo-radiation with temozolomide. J Clin Oncol 2007;25(18S):2009.

15. Brix G, Zaers J, Adam LE, et al. Performance evaluation of a whole-body PET scanner using the NEMA protocol. National Electrical Manufacturers Association. J Nucl Med 1997;38(10):1614-23. [PubMed: 9379202]

16. Torii K, Tsuyuguchi N, Kawabe J, et al. Correlation of amino-acid uptake using methionine PET and histological classifications in various gliomas. Ann Nucl Med 2005;19(8):677-83. [PubMed: 16444993]

17. Cao Y. Development of Image Software Tools for Radiation Therapy Assessment. Medical Physics 2005;32:2136.

18. Lee SW, Fraass BA, Marsh LH, et al. Patterns of failure following high-dose 3-D conformal radiotherapy for high-grade astrocytomas: a quantitative dosimetric study. Int J Radiat Oncol Biol Phys 1999;43(1):79-88. [PubMed: 9989517]

19. Park I, Tamai G, Lee MC, et al. Patterns of recurrence analysis in newly diagnosed glioblastoma multiforme after three-dimensional conformal radiation therapy with respect to pre-radiation therapy magnetic resonance spectroscopic findings. Int J Radiat Oncol Biol Phys. 2007

20. Pirzkall A, Li X, Oh J, et al. 3D MRSI for resected high-grade gliomas before RT: tumor extent according to metabolic activity in relation to MRI. Int J Radiat Oncol Biol Phys 2004;59(1):126-37. [PubMed: 15093908]

21. Pirzkall A, McKnight TR, Graves EE, et al. MR-spectroscopy guided target delineation for highgrade gliomas. Int J Radiat Oncol Biol Phys 2001;50(4):915-28. [PubMed: 11429219]

22. Cao Y, Nagesh V, Hamstra D, et al. The extent and severity of vascular leakage as evidence of tumor aggressiveness in high-grade gliomas. Cancer Res 2006;66(17):8912-7. [PubMed: 16951209]

23. Hamstra DA, Chenevert TL, Moffat BA, et al. Evaluation of the functional diffusion map as an early biomarker of time-to-progression and overall survival in high-grade glioma. Proc Natl Acad Sci U S A 2005;102(46):16759-64. [PubMed: 16267128] 


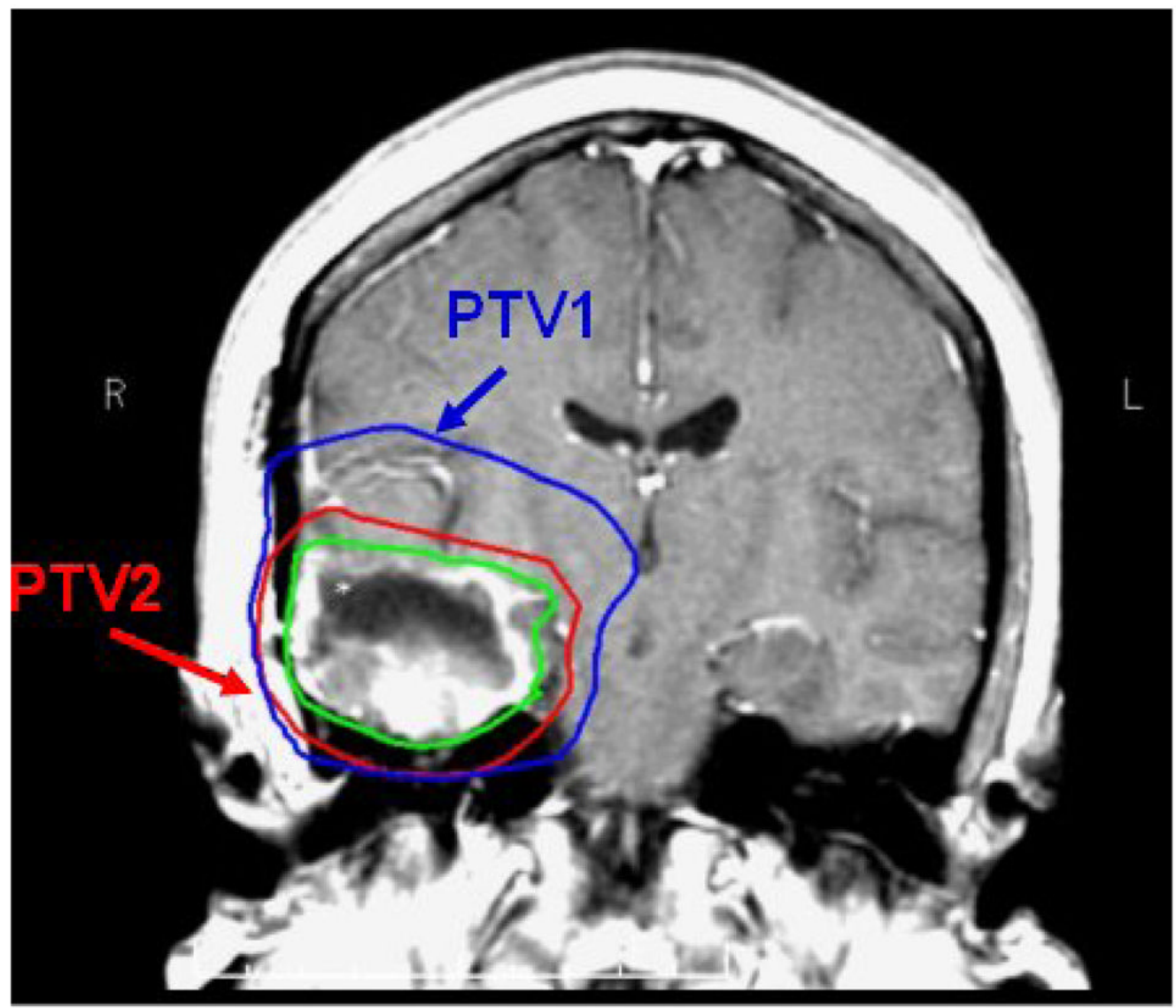

FIGURE 1.

Coronal slice of T1 post-gadolinum MRI with GTV outlined in green. PTV1, shown in blue, receives $60 \mathrm{~Gy}$ in 30 fractions. PTV2, shown in red, receives protocol dictated dose over the same 30 fractions using a conformal simultaneous IMRT boost. 


\section{PET vol by resection}

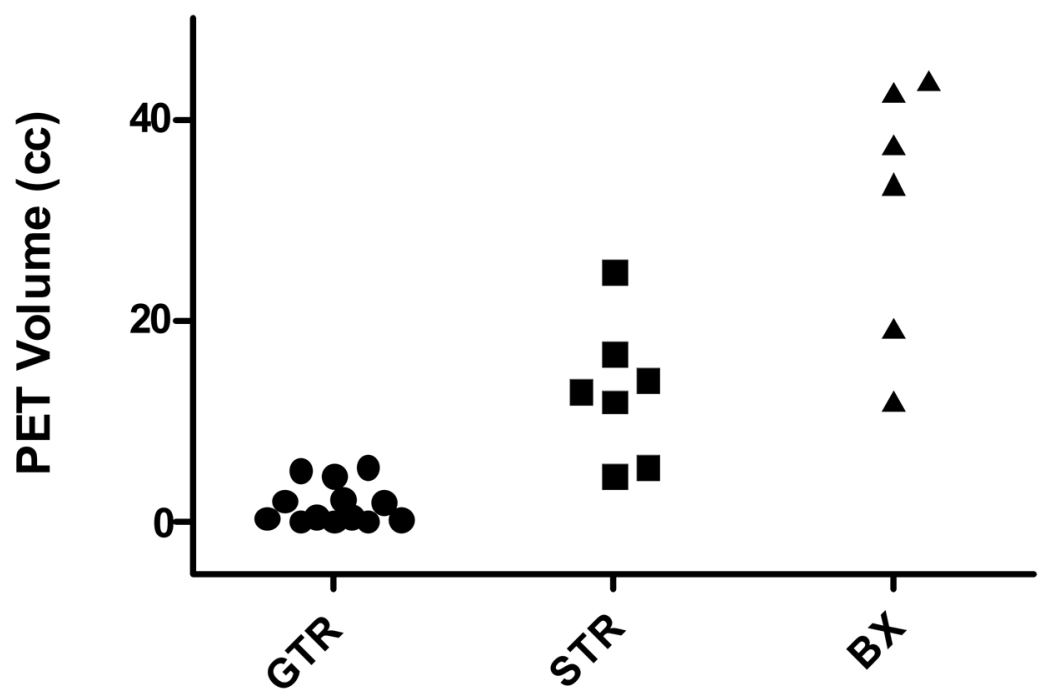

FIGURE 2.

MET-PET volume sorted by extent of surgery. 
FIGURE 3A.
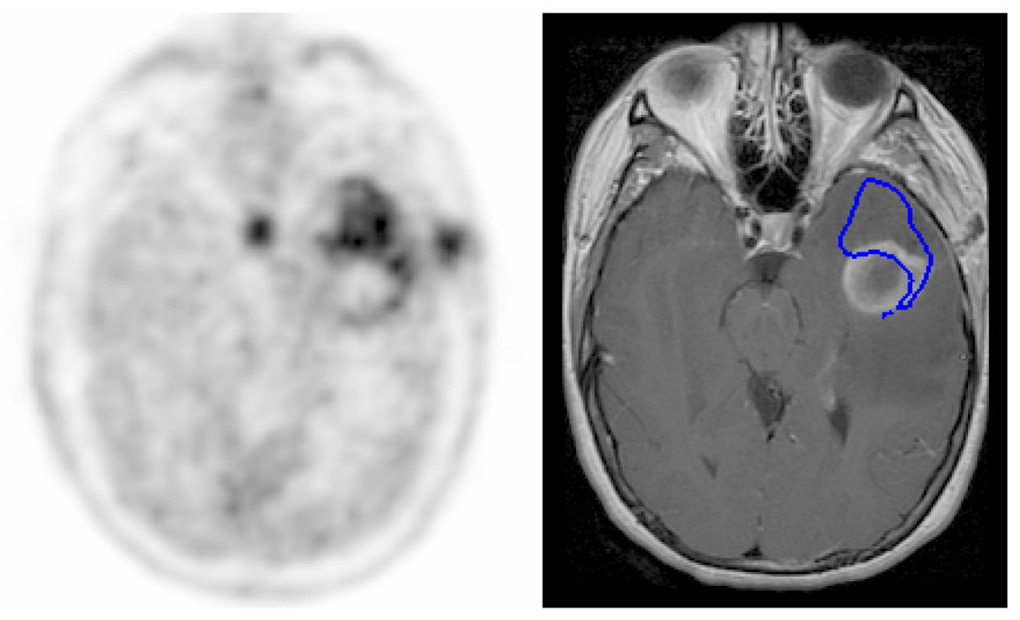

FIGURE $3 B$.
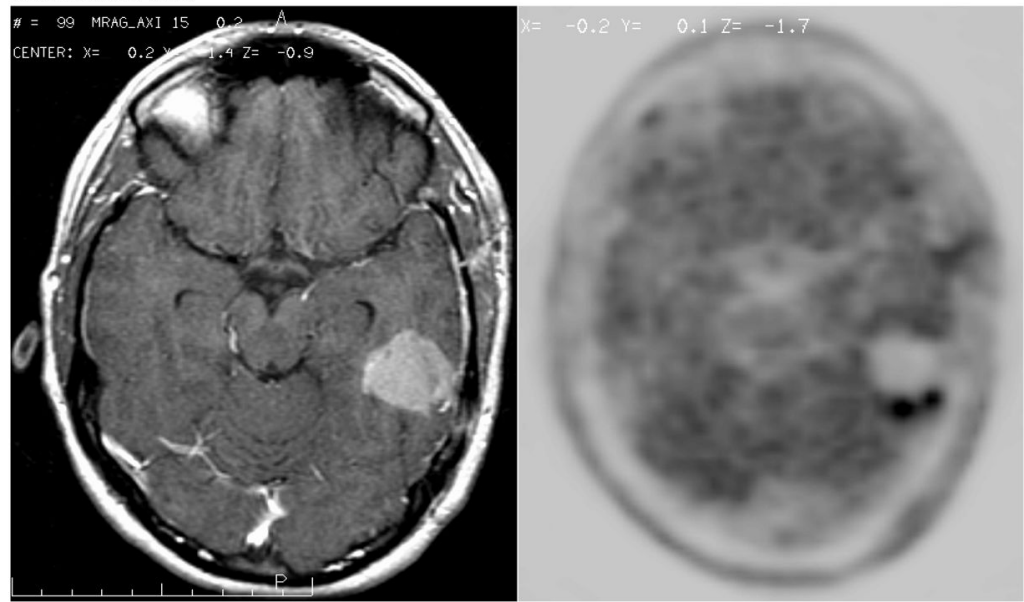

FIGURE 3.

FIGURE 3A. MET-PET (left) and Gd-enhanced T1-weighted MRI (right) after stereotactic biopsy. PET-GTV (outlined in blue on MRI) extends beyond the contrast-enhancing lesion. FIGURE 3B. Post-operative Gd-enhanced T1-weighted MRI (left) demonstrates resection cavity with no evidence of residual disease, but 11C Methionine PET scan (right) demonstrates an area of residual tumor that was not appreciated on the MRI. 


\section{FIGURE 4A}
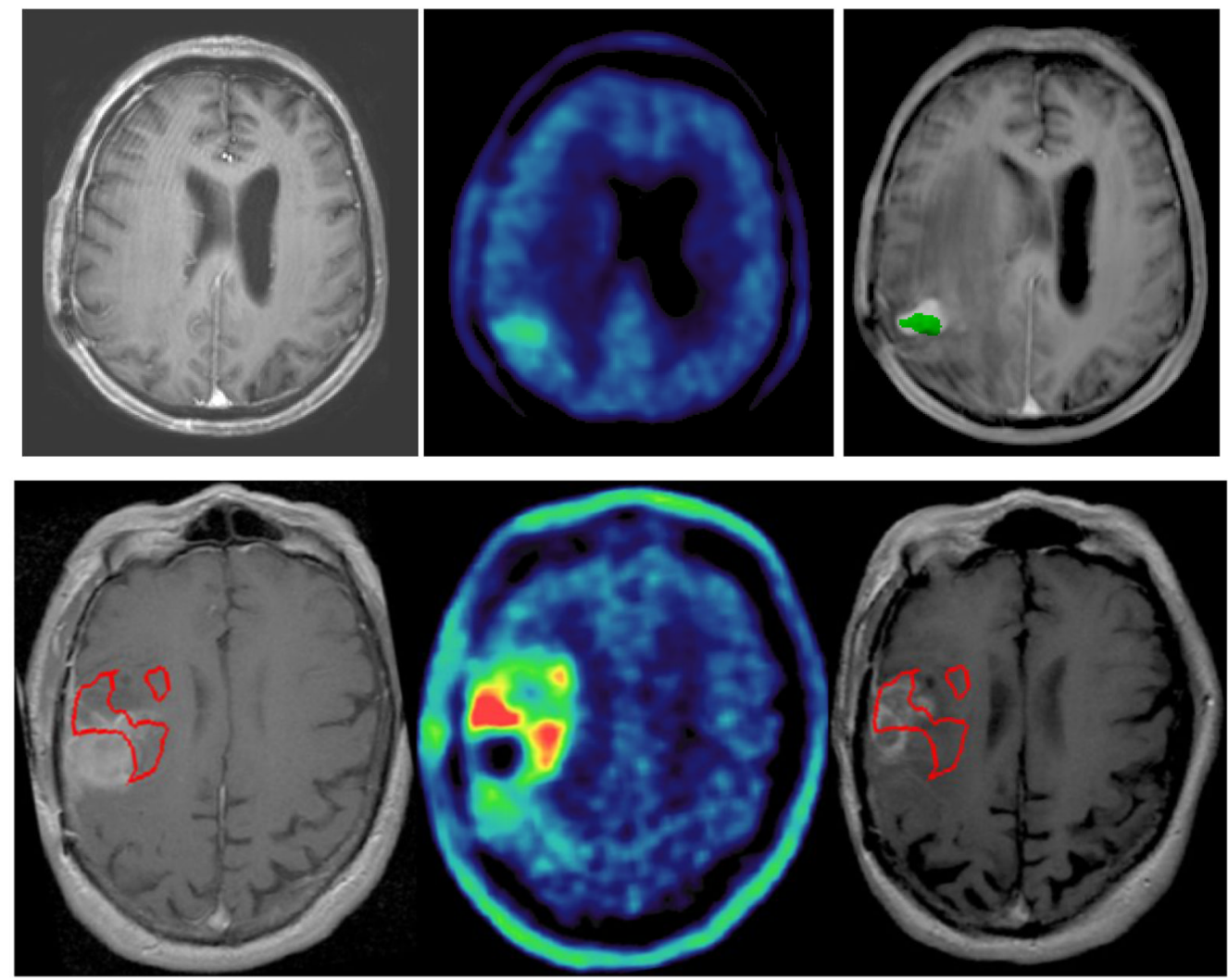

FIGURE 4.

FIGURE 4A. A patient with a distinct nodule noted on 11C MET PET that was not fully encompassed in the RT plan. Gadolinium-enhanced, T1-weighted MRI (left) shows no appreciable contrast enhancing lesion in the corresponding area of high uptake in the right parietal region on pre-treatment MET-PET (center). MRI one month after completion of therapy (right) shows a new contrast-enhancing nodule in the area of initial MET PET uptake (green). The subsequent clinical course confirmed disease progression.

Figure 4B: Images from a representative patient with a PET-GTV extending beyond the contrast-enhancing lesion on MRI. Pre-treatment gadolinium-enhanced, T1-weighted MRI (left) shows enhancement along the resection cavity. There is a focus of increased MET-PET (center) uptake directly above the resection cavity. Subsequent MRI including MR

Spectroscopy and PET imaging performed 10 months following completion of therapy shows tumor progression present above the resection cavity which overlaps with, but is not fully encompassed by, the PET-GTV. 
Patient Characteristics $(\mathrm{n}=26)$

Table 1

\begin{tabular}{|lc|}
\hline Age, median (range) & $53(20-75)$ \\
KPS & 23 \\
$90-100$ & 2 \\
80 & 1 \\
70 & \\
RPA classification[19] & 11 \\
III & 11 \\
IV & 4 \\
V & 1 \\
Radiation Prescription Dose & \\
66 Gy & 3 \\
72 Gy & $7^{*}$ \\
75 Gy & 7 \\
78 Gy & 8 \\
81 Gy & \\
Extent of Surgery & 13 \\
Gross total resection & 7 \\
Subtotal resection & 6 \\
Biopsy only & \\
\hline
\end{tabular}

* 1 patient received only $20 \mathrm{~Gy}$ 
Table 2

Patterns of Failure and PET-GTV Coverage

\begin{tabular}{|lccc|}
\hline & Central & In-field & Marginal \\
$>$ 95\% PET-GTV within 95\% IDS & 9 & 1 & 1 \\
$<$ <95\% PET-GTV within 95\% IDS & 0 & 2 & 3 \\
\hline
\end{tabular}

$\mathrm{P}=0.004$ (Fisher exact test) 